\title{
Therapeutic drug monitoring in drug overdose
}

\author{
Andrew H. Dawson \& lan M. Whyte \\ Department of Clinical Toxicology and Pharmacology, Newcastle Mater Hospital, Locked Bag 7, Hunter Regional Mail Centre, NSW 2310, Australia
}

\begin{abstract}
The treatment of poisoned patients is still largely defined by history, clinical assessment and interpretation of ancillary investigations. Measurement of drug concentrations is clinically important for relatively few compounds. Most measurements form an adjunct to and should not be considered a substitute for clinical assessment. Drug concentrations are particularly important for those compounds where the concentration is predictive of serious toxicity in an otherwise asymptomatic patient.
\end{abstract}

Keywords: drug monitoring, overdose, poisoned patient, toxicity

\section{Introduction}

In clinical toxicology, the measurement of drug concentrations can fulfil a research or a clinical purpose. The technology is available to assay virtually any drug or toxin in most body fluids. However, in the vast majority of patients presenting with overdose, there is no need for routine measurement of drug concentrations or drug screens. In those patients where the history is unclear, selective assaying of drugs should be guided by knowledge of the local epidemiology of poisoning, clinical examination and the interpretation of other investigations. There are very few drugs for which a blood level alone dictates management decisions.

\section{Research}

The use of drug concentrations for research in toxicology is both important and problematic. An understanding of the kinetics of drugs taken in overdose may contribute to the development of more rational treatment and improve clinical outcomes. The problems in assessing such data relate to the variables inherent in the clinical presentation. Such variables include ingested doses, times to presentation, gastrointestinal decontamination and the likelihood that in most patients there is an opportunity to collect only very few samples. The problems are exaggerated by potential ethical difficulties in collecting blood from impaired patients for tests which may not have direct bearing on that individual's clinical outcome. Such difficulties may be minimized by pooling data from multiple patients (e.g. MEMO Project at

Correspondence: Dr A. H. Dawson, Department of Clinical Toxicology and Pharmacology, Newcastle Mater Hospital, Locked Bag 7, Hunter Regional Mail Centre, NSW 2310, Australia. E-mail: mdahd@cc.newcastle.edu.au.
www.ctlu.se/CTLU-MEMO.html) and using a population based pharmacokinetic approach.

\section{Screening}

In general the cost and the often slow turn around time of undifferentiated screens to detect the presence of toxins rarely alters clinical outcomes. Screening may have an important role in the epidemiological assessment of poisoning as it is a common finding that more substances are detected in urine than are recorded from the history. Specific qualitative tests (e.g. amatoxins, paraquat) may be of clinical assistance in determining evidence of exposure. Clinicians are better served by relying on a careful interpretation of the history and clinical examination in conjunction with readily accessible investigations such as ECG, electrolytes and acid-base analysis.

\section{Methods}

The principal methods utilized to measure drug concentrations in clinical toxicology are the same as those used in therapeutic drug monitoring. These include fluorescent immunoassay, enzyme immunoassay, thin-layer chromatography, high performance liquid chromatography, gas chromatography, mass spectroscopy, flame photometry and simple colorimetric methods.

\section{Specific drugs}

The main purpose of this article is to discuss those compounds where measurement of drug concentration is clinically indicated. To be useful clinically a drug concentration should assist in one or more of the following areas: diagnosis, prognosis, guiding therapy or assessing the efficacy of current therapy. Even when these 
criteria are satisfied the drug concentration needs to be interpreted in the context of the individual patient's clinical condition and other factors which may influence the pharmacodynamic response to any blood level (such as coexistent disease or age).

Using these criteria the list of drugs for which blood concentrations are clinically useful is relatively small. Individual drugs are discussed in diminishing order of evidence of clinical importance.

\section{Paracetamol}

Paracetamol concentrations impart diagnostic and prognostic information and define the need for therapy in overdose. The prognostic importance of a serum paracetamol concentration in predicting hepatotoxicity is well established [1, 2]. For single ingestions of paracetamol, taken at a known time, an appropriately timed blood level (between 4 and 15 h) can be plotted on a nomogram which reliably predicts those patients at risk of hepatotoxicity [3]. The nomogram (see Figure 1) reflects saturation of conjugative pathways of paracetamol metabolism and describes a group of patients whose paracetamol half-life is greater than $4 \mathrm{~h}$. The nomogram is only validated for the times between 4 and $15 \mathrm{~h}$ after a single ingestion. Theoretically, as paracetamol concentration falls, a point is reached where metabolism is no longer saturated, the half-life becomes shorter and it is possible that concen-

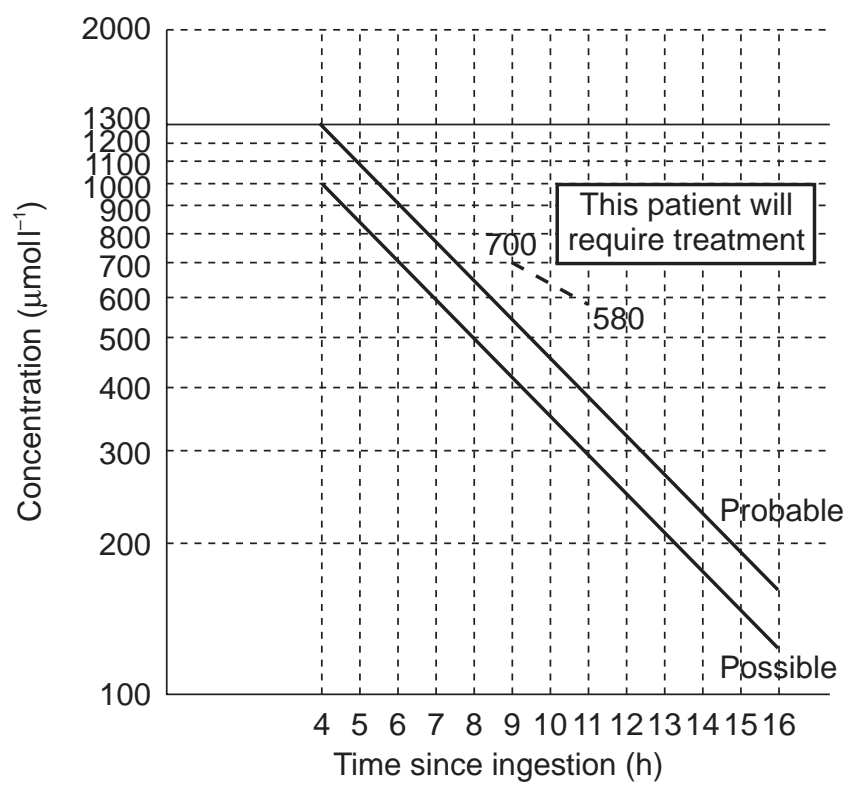

Figure 1 Paracetamol nomogram (Modified from Rumack et al. [27]. The line commencing from $1300 \mu \mathrm{mol} \mathrm{l}^{-1}$ at $4 \mathrm{~h}$ defines those patients who require treatment with $\mathrm{N}$-acetylcysteine. The slope of the line reflects a half-life of $4 \mathrm{~h}$.

If the time of ingestion is not known then plotting a pair of samples allows an estimate of whether that patient is at risk of hepatotoxicity. In this example the slope of the line reflects a half-life which is greater than $4 \mathrm{~h}$. trations below an extrapolation of the line beyond $15 \mathrm{~h}$ may still be associated with significant hepatotoxicity. The height of the concentration above the line correlates with an increasing risk of hepatotoxicity and death. Patients whose paracetamol concentration is above the line between $300 \mathrm{mgl}^{-1}\left(1985 \mu \mathrm{moll}^{-1}\right)$ at $4 \mathrm{~h}$ and $45 \mathrm{mgl}^{-1}\left(300 \mathrm{moll}^{-1}\right)$ at $15 \mathrm{~h}$ have a $90 \%$ risk of hepatotoxicity. In contrast, concentrations between a parallel line starting at $200 \mathrm{mg} \mathrm{l}^{-1}\left(1325 \mu \mathrm{mol} \mathrm{l}^{-1}\right)$ and the $300 \mathrm{mg}^{-1}$ line have a risk of hepatotoxicity of $25 \%$ [1].

Paracetamol concentrations should be assessed in all patients who have ingested a potentially dangerous dose of paracetamol or if there is reasonable clinical suspicion that this has occurred. The sample should be taken at least $4 \mathrm{~h}$ after ingestion at which time paracetamol has generally been fully absorbed. While backwards extrapolation of the nomogram line from $4 \mathrm{~h}$ to $0 \mathrm{~h}$ is not reliable in predicting toxicity, patients whose concentrations are substantially below the line in this early phase, are unlikely to develop toxicity [4]. Attempts have been made to allow for patients at increased risk of hepatotoxicity due to pre-existing glutathione depletion or enzyme induction (e.g. chronic alcohol abuse) by using a lower treatment line [5]. The selection of this lower line has been arbitrary with various lines starting at $150 \mathrm{mgl}^{-1}$ $\left(1000 \mu \mathrm{mol} \mathrm{l}^{-1}\right)$ or $100 \mathrm{mg} \mathrm{l}^{-1}\left(660 \mu \mathrm{mol} \mathrm{l}^{-1}\right)$. The evidence for the need for a lower line is based on case reports only.

The nomogram is not valid for multiple ingestions or ingestions of controlled release paracetamol. In these situations, a conservative threshold for commencing treatment would be the ingestion of more than $150 \mathrm{mg} \mathrm{kg}^{-1}$ of paracetamol within a $24 \mathrm{~h}$ period. Difficulties also arise when the time of ingestion is not known. In these circumstances our practice is to attempt a worst case analysis and treat on this basis and then repeat the paracetamol level at least $2 \mathrm{~h}$ after the previous collection. If the two concentrations are then plotted on the nomogram and the fall in concentration is steeper than the treatment threshold line then toxicity is unlikely (Figure 1). Alternatively, the half-life can be calculated using the formula.

$$
t_{1 / 2}=0.693 \star \frac{(t 2-t 1)}{(\ln C 1-\ln C 2)}
$$

Where $C 1$ is the concentration at time $t 1$ and $C 2$ is the concentration at time $t 2$ after ingestion. To have any clinical utility the time intervals between samples must be relatively short $(2-4 \mathrm{~h})$. In this setting it has been suggested that the coefficient of variation for the assay is such that three measurements are required for accurate calculation of half-life [6]. Allowing for a $5 \%$ error in the assay, toxicity is unlikely in patients whose measured 
half-life is less than $3 \mathrm{~h}$ when calculated using paired samples at least $2 \mathrm{~h}$ apart.

Patients who are at risk of hepatotoxicity should receive intravenous $N$-acetylcysteine [1, 7]. Ideally treatment should be commenced within $8 \mathrm{~h}$ of ingestion as it is completely protective at that time. Patients who present after $8 \mathrm{~h}$ with a significant poisoning (>150 $\mathrm{mg} \mathrm{kg}^{-1}$ ingestion) should have $N$-acetylcysteine commenced prior to the return of the paracetamol concentration. In these patients the need for ongoing treatment can be defined by the paracetamol concentration.

\section{Theophylline}

Theophylline concentrations impart diagnostic and prognostic information, define the need for and measure the efficacy of therapy in overdose. Theophylline concentrations predict the risk of seizures and cardiac toxicity in both acute and chronic toxicity [8, 9]. As there is often a lag time between achieving potentially toxic concentrations and having a major complication it has been suggested that these concentrations should be used as an indication for extracorporal removal (charcoal haemoperfusion or haemodialysis) $[10,11]$. The concentrations of theophylline associated with major toxicity differ for acute single ingestions $\left(100 \mathrm{mg} \mathrm{l}^{-1}\right.$ or $\left.550 \mu \mathrm{mol} \mathrm{l}^{-1}\right)$ and chronic ingestions $\left(40 \mathrm{mg} \mathrm{l}^{-1}\right.$ or $\left.220 \mu \mathrm{moll}^{-1}\right)$. The difference in toxic concentration between the two groups probably reflects differences in the rate of accumulation and absolute concentration of intracellular cyclic AMP. Patients with pre-existing neurological or cardiovascular disease or at the extremes of age have increased organ sensitivity to theophylline and may develop major complications at lower concentrations [11].

Patients who have ingested more than $10 \mathrm{mg} \mathrm{kg}^{-1}$ of theophylline should receive repeated doses of activated charcoal. Activated charcoal reduces ongoing absorption and enhances elimination by gastrointestinal dialysis [11]. The majority of theophylline poisoning cases are due to ingestion of controlled release forms of medication. This leads to a prolonged absorption phase and a peak concentration that can be delayed for up to $24 \mathrm{~h}$. For this reason theophylline concentrations should be measured on admission and at $2 \mathrm{~h}$ intervals thereafter until theophylline levels are clearly falling [12]. This allows the clinician to assess the efficacy of gastrointestinal decontamination and provides a reasonable window of opportunity to institute extracorporal removal if gastrointestinal decontamination is unsuccessful.

\section{Digoxin}

Digoxin concentrations impart diagnostic and prognostic information and can define both the need for therapy in overdose and the dose of digoxin antibodies (Fab) required [13-15]. In samples taken within $6 \mathrm{~h}$ of digoxin ingestion the concentrations will be spuriously high as digoxin is still in its distribution phase. Samples taken after $6 \mathrm{~h}$ will allow a more accurate estimate of the body's digoxin burden thereby allowing more accurate calculation of digoxin antibody dose. Following administration of Fab fragments, digoxin moves from tissue compartments into the blood. This is reflected by an increase in measured digoxin concentration because most assays measure digoxin bound to the Fab fragment. For this reason measuring digoxin concentrations after the administration of Fab fragments is pointless unless free digoxin is measured by ultrafiltration.

In the majority of patients with digoxin toxicity the need for specific treatment is dictated by the occurrence of life threatening dysthrythmia, significant cardiac compromise or uncontrolled hyperkalaemia rather than the digoxin concentration alone. In these circumstances digoxin antibodies can be life saving. However, in the absence of major clinical toxicity, an absolute digoxin level of greater than $10 \mathrm{nmol}^{-1}$ taken $6 \mathrm{~h}$ after the last dose is an indication for the use of digoxin Fab fragments. In this setting the use of Fab fragments is more cost effective than prolonged monitoring.

Digoxin Fab dose can be determined by titrating incremental doses against clinical manifestations of digoxin toxicity. Alternatively, in acute ingestions, a Fab dose can be calculated from the dose of digoxin ingested. Probably the most accurate method of determining Fab dose is by using the digoxin concentration to calculate the total body digoxin load and then calculate an appropriate Fab dose $(1 \times 38 \mathrm{mg}$ vial of digoxin Fab fragments binds to $0.5 \mathrm{mg}$ of digoxin).

This calculation uses the average volume of distribution (adults $7-81 \mathrm{~kg}^{-1}$, neonates $101 \mathrm{~kg}^{-1}$, infants $161 \mathrm{~kg}^{-1}$ ) and the measured digoxin concentration.

Total body burden of digoxin $=$ concentration in $\mu \mathrm{g} 1^{-1} \quad$ (or nmol $\left.1^{-1} / 1.28\right) \times$ weight $(\mathrm{kg}) \times$ volume of distribution.

Number of $38 \mathrm{mg}$ ampoules of Fab required $=$ Total digoxin body burden/0.5.

\section{Iron}

Iron concentrations collected at an appropriate time impart diagnostic and prognostic information and help define therapy in overdose [16, 17]. Following poisoning with iron, toxicity develops when serum iron concentrations exceed the iron binding capacity of transferrin in blood. The free circulating iron damages many organs by direct cytotoxicity, effects on vasculature and release of inflammatory mediators. Iron concentrations taken within $4-6 \mathrm{~h}$ of ingestion of normal release preparations give 
good prognostic information for the chances of multi organ toxicity and guide therapy (see Table 1). Patients presenting with late iron poisoning $(>6 \mathrm{~h})$ may have serum iron concentrations within the normal range as most of the iron has moved into the tissues. In this situation the indication for chelation treatment is based on the presence and severity of clinical toxicity. Clinical toxicity includes severe haemorrhagic gastroenteritis, circulatory collapse, acidosis, lethargy, coma and convulsions and hepatic or renal failure.

\section{Lithium}

Depending on the clinical type of presentation, lithium concentrations are useful for diagnosis and defining prognosis [18, 19]. The calculation of renal lithium clearance assists in defining which patients are most likely to benefit from haemodialysis. As lithium clearance during haemodialysis is in the order of 100$120 \mathrm{ml} \mathrm{min}^{-1}, 4 \mathrm{~h}$ of haemodialysis is equivalent to a $24 \mathrm{~h}$ clearance of $16-20 \mathrm{ml} \mathrm{min}^{-1}$. Thus, patients whose intrinsic renal lithium clearance is greater than $20 \mathrm{ml} \mathrm{min}^{-1}$ generally do not require haemodialysis.

Formula: $\quad \mathrm{Li} \quad$ clearance $=($ urinary lithium concentration $\times$ urine flow $\left.\left(\mathrm{ml} \mathrm{min}^{-1}\right)\right) /$ serum Li concentration.

e.g. In a $2 \mathrm{~h}$ urine collection the patient passes $480 \mathrm{ml}$ urine (i.e. $4 \mathrm{ml} \mathrm{min}^{-1}$ ) with a urinary lithium concentration of $21 \mathrm{mmol}^{-1}$. The patient's serum Li concentration is $3.0 \mathrm{mmoll}^{-1}$. Renal $\mathrm{Li}$ clearance is = $\left(21 \mathrm{mmol} \mathrm{l}^{-1} \times 4 \mathrm{ml} \mathrm{min}^{-1}\right) / 3 \mathrm{mmol} \mathrm{l}^{-1}=28 \mathrm{ml} \mathrm{min}^{-1}$

As the fractional lithium clearance is $20-30 \%$ of creatinine clearance a formal calculation of lithium clearance is not needed in patients whose measured or calculated creatinine clearance is less than $60 \mathrm{ml} \mathrm{min}^{-1}$. These patients need haemodialysis.

Steady state plasma lithium concentrations are normally measured at $12 \mathrm{~h}$ after the last dose. The equilibration of lithium between plasma and brain is extremely slow. For this reason a clinical distinction should be made between the three types of presentation of lithium poisoning. These are:

Table 1 Clinical significance of iron concentrations.

\begin{tabular}{|c|c|}
\hline Serum iron concentrations & Clinical significance \\
\hline $10-30 \mu \mathrm{mol} \mathrm{l}^{-1}$ & Normal range \\
\hline $30-60 \mu \mathrm{mol} \mathrm{l}^{-1}$ & Significant toxicity is unlikely \\
\hline $60-90 \mu \mathrm{mol} \mathrm{l}^{-1}$ & $\begin{array}{l}\text { Possible toxicity. Symptomatic patients } \\
\text { should receive desferrioxamine }\end{array}$ \\
\hline Greater than $90 \mu \mathrm{mol} \mathrm{l}^{-1}$ & $\begin{array}{l}\text { Intravenous desferrioxamine therapy } \\
\text { indicated irrespective of clinical state }\end{array}$ \\
\hline
\end{tabular}

1 Acute large ingestions of lithium, in patients not taking lithium chronically.

2 Acute large ingestions on a background of chronic lithium treatment which may or may not produce toxicity.

3 Chronic lithium toxicity which is frequently symptomatic.

Acute ingestions in the absence of previous lithium therapy result in high concentrations but are rarely associated with severe toxicity. Those patients on chronic therapy presenting with an acute ingestion are more likely to develop clinical toxicity as their brain has a significant lithium load.

In acute ingestions a serum lithium level may confirm ingestion but does not correlate with clinical signs because there has been insufficient time for equilibration with the brain. However, in chronic toxicity, equilibration has occurred and there is good correlation between serum lithium concentrations and clinical signs (see Table 2). Any patient with central nervous system symptoms or lithium concentrations of greater than $1.5 \mathrm{mmoll}^{-1}$ should be admitted.

Haemodialysis should be considered in patients who have renal impairment (calculated creatinine clearance $<60 \mathrm{ml} \mathrm{min}^{-1}$ ) or demonstrate low renal clearance of lithium and signs of severe clinical toxicity. All patients require careful attention to sodium and fluid balance. As clearance from the plasma compartment is much faster than from the CNS it is not uncommon for patients who have presented with chronic lithium toxicity to still have persistent signs of CNS toxicity when lithium concentrations have fallen into or below the therapeutic range.

In those patients who undergo haemodialysis it is common for a rebound increase in lithium concentrations to occur $6-12 \mathrm{~h}$ after ceasing haemodialysis. This is a consequence of the relatively slow equilibration between tissue and plasma compartments. Such patients therefore should have repeat serum lithium concentrations to determine if further dialysis is indicated. Dialysis is usually continued until the rebound lithium concentration is $<1 \mathrm{mmol} \mathrm{l}^{-1}$.

Table 2 Relationship between lithium concentration and clinical effects.

Chronic lithium concentration and toxicity

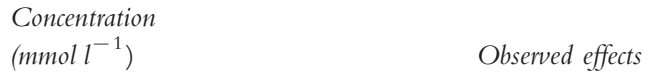

\begin{aligned} & \hline 0.5 None \\ & 1.0 Mild tremor \\ & 1.5 Coarse tremor \\ & 2.0 Hyperreflexia, dysarthria \\ & 2.5 Myoclonic and other involuntary movements \\ & Ataxia and confusion \\ &$>3.0$ Marked delirium, coma, seizures \\ & \hline\end{aligned}




\section{Salicylate}

Salicylate concentrations impart diagnostic and prognostic information and define therapy in overdose [20-22]. Clinical toxicity is primarily a function of the degree and duration of salicylate induced acid base disturbance. Significant clinical toxicity is apparent in chronic ingestions at lower serum levels than in acute ingestions.

Salicylate concentrations should be measured in any patient who has a history of potentially serious ingestion $\left(>100 \mathrm{mg} \mathrm{kg}^{-1}\right.$ ) or has a significant electrolyte or acidbase disturbance after salicylate ingestion. In addition to clinical signs of severe intoxication and the measurement of acid-base disturbance, salicylate concentrations can define treatment. The concentrations are predictive of severity and give some indication of the need for intensive care admission and haemodialysis (see Table 3). Concentrations measured in samples taken within $6 \mathrm{~h}$ of ingestion can be interpreted using a nomogram [23].

Repeated salicylate concentrations also give some measure of the effectiveness of gastrointestinal decontamination and elimination enhancement. Salicylates may form concretions or bezoars leading to a prolonged and episodic absorptive phase. It is necessary to take repeat concentrations $2 \mathrm{~h}$ apart until it is demonstrated that the level is falling.

\section{Toxic alcohols}

In practice, the management of toxic alcohol ingestions is dictated by clinical signs, acid-base and osmolarity measurements. Metabolic acidosis results from accumulation of acid metabolites of ethylene glycol or methanol and from promotion of lactic acid formation due to reduced NAD/NADH ratios. This metabolic disturbance may be reflected by an anion gap $>14 \mathrm{mmol} \mathrm{l}^{-1}$

$$
\left(\text { Anion gap }=\mathrm{Na}-\left(\mathrm{HCO}_{3}+\mathrm{Cl}\right)\right) .
$$

An osmolal gap $>17 \mathrm{mmoll}^{-1}$ is suggestive of the presence of toxic alcohols. The osmolal gap is the difference between measured and calculated osmolality.

Calculated osmolality $=2 \quad \mathrm{Na}+$ Glucose + urea (all in $\mathrm{mmol}^{-1}$ ).

The measured osmolality should be done by freezing point depression (other methods are too inaccurate). In

Table 3 Salicylate concentrations and clinical implications.

\begin{tabular}{ll}
\hline Salicylate concentrations & Clinical implications \\
\hline$>4 \mathrm{mmol} \mathrm{l}^{-1}\left(65 \mathrm{mg}^{-1}\right)$ & ICU monitoring \\
$>9.4 \mathrm{mmol}^{-1}$ in acute ingestions & Haemodialysis \\
$>4.45 \mathrm{mmol}^{-1}$ in chronic & Haemodialysis \\
intoxication & \\
\hline
\end{tabular}

late presentations the osmolar gap may be normal as the alcohols have been metabolized.

\section{Ethylene glycol}

An ethylene glycol concentration of $>50 \mathrm{mg} \mathrm{dl}^{-1}$ is a possible indication for haemodialysis and a definite indication for 4-methylpyrazole (4MP) or ethanol infusion [24]. However, as the toxicity of ethylene glycol is due to its metabolic products it is possible patients may have significant toxicity with low or undetectable ethylene glycol concentrations. In this setting, clinical signs and metabolic disturbance are a more accurate reflection of toxicity.

\section{Methanol}

A methanol level of greater than $50 \mathrm{mg} \mathrm{dl}^{-1}$ is a possible indication for haemodialysis and a definite indication for $4 \mathrm{MP}$ or ethanol infusion. Patients with methanol toxicity who present late may have relatively low methanol concentrations, the clinical toxicity being due to methanol metabolites.

\section{Ethanol}

Ethanol concentrations correlate well with clinical toxicity in alcohol naive patients but tolerance can be extreme in patients with regular consumption. In overdose, their use is mainly diagnostic. While haemodialysis can increase the clearance of ethanol, it is rarely required. Indications for its use should be based on clinical severity rather than an absolute concentration.

\section{Anticonvulsants}

Measurement of anticonvulsant concentrations will confirm ingestion but do not substantially influence treatment in overdose, which is supportive care. In overdoses of controlled release preparations a persitently rising concentration suggests inadequate decontamination and may lead to consideration of endoscopy or whole bowel irrigation [12].

\section{Paraquat}

In clinical practice, paraquat concentrations are useful for confirming ingestion and defining prognosis but do not influence treatment, which is predominantly supportive care $[25,26]$. The only definitive treatment for paraquat poisoning is prompt and effective gastrointestinal decontamination. While charcoal haemoperfusion commenced within $2 \mathrm{~h}$ of ingestion may alter clinical outcome, it is rare that patients present and have charcoal haemoperfusion commenced within that period [23]. A plasma 


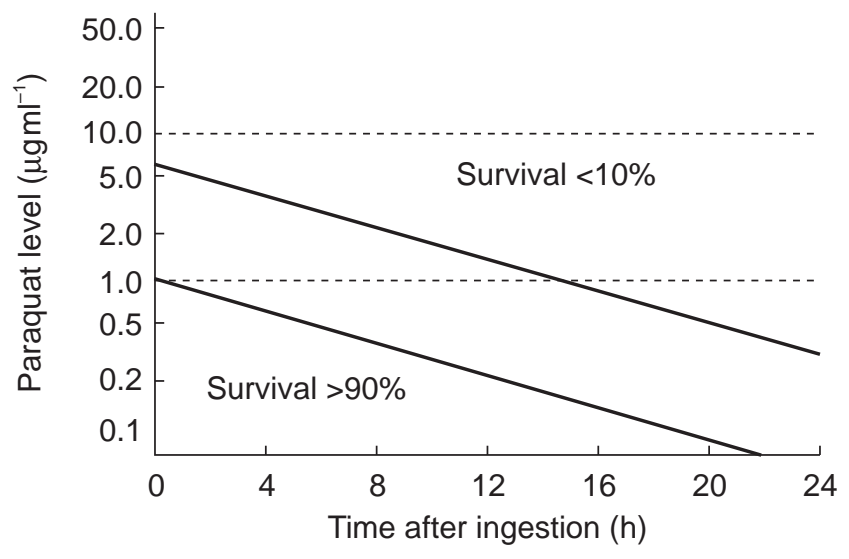

Figure 2 Paraquat nomogram (modified from Hart et al. [26].

concentration of greater than $5 \mu \mathrm{g} \mathrm{ml}^{-1}$ at any time following ingestion invariably indicates a fatal outcome. The blood concentrations can be interpreted on a nomogram (see Figure 2) to indicate the likelihood of survival. A simple qualitative colorimetric test of the urine can be performed which, if positive, indicates paraquat exposure but does not define prognosis. If this test is negative on urine passed 2-6 h after exposure then significant toxicity is unlikely.

\section{References}

1 Prescott LF. Paracetamol (Acetaminophen) A critical bibliographic review. Publisher, Taylor \& Francis 1996.

2 Prescott LF, Wright N, Roscoe P, Brown S. Plasma Paracetamol half life and hepatic necrosis in patients with paracetamol overdosage. Lancet 1971; i: 519-522.

3 Rumack BH, Mathew $\mathrm{H}$. Acetaminophen poisoning and toxicity. Paediatrics $1975 ; \mathbf{5 5}$ : 871-876.

4 Cannavo J, Hung O, Nelson L, Hoffman RS. A mathematical model highlighting the limitations of a two point acetaminophen half life. Clin Toxicol 1997; 35: 510.

5 Smith JAE, Hine ID, Beck P, Routledge PA. Paracetamol toxicity is enzyme induction important? Human Toxicol 1986; 5: 383-385.

6 Douglas DR, Smilkenstein MJ, Rumack BH. APAP levels within 4 hours: are they useful? Vet Hum Toxicol 1994; 36: 356.

7 Janes J, Routledge PA. Recent developments in the management of paracetamol (acetaminophen) poisoning. Drug Safety 1992; 7: 170-177.

8 Paloucek FP, Rodvold KA. Evaluation of theophylline overdoses and toxicities. Ann Emerg Med 1988; 17: 135-144.
9 Olson KR, Benowitz NL, Woo OF, Pond SM. Theophylline Overdose: acute single ingestion versus chronic repeated overmedication. Am J Emerg Med 1985; 3: 386-394.

10 Woo OF, Pond SM, Benowitz NL, Olson KR. Benefit of haemoperfusion in acute theophylline intoxication. Clin Toxicol 1984; 22: 411-442.

11 Dawson AH, Whyte IM. Treatment of theophylline toxicity. Med J Australia 1989; 151: 689-693.

12 Buckley NA, Dawson AH, Reith DA. Controlled release drugs in overdose, clinical considerations. Drug Safety 1995; 12: 73-84.

13 Marchlinski FE, Hook BG, Callans DJ. Which cardiac disturbances should be treated with digoxin immune $\mathrm{Fab}$ (ovine) antibody? Am J Emergency Med 1991; 9: 24-28.

14 Wells TG, Young RA, Kearns GL. Age-related differences in digoxin toxicity and its treatment. Drug Safety 1992; 7: $135-151$.

15 Woolf AD, Wenger TL, Smith TW, Lovejoy FH Jr. Results of multicenter studies of digoxin-specific antibody fragments in managing digitalis intoxication in the pediatric population. Am J Emergency Med 1991; 9: 16-20.

16 Mills KC, Curry SC. Acute iron poisoning. Emergency Med Clinics North America 1994; 12: 397-413.

17 Tenenbein M. Benefits of parenteral deferoxamine for acute iron poisoning. J Toxicol-Clin Toxicol 1996; 34: 485-489.

18 Amdisen A. Clinical features and management of lithium poisoning. Med Toxicol 1988; 3: 18-32.

19 Jaeger A, Sauder P, Kopferschmitt J, et al. When should dialysis be performed in lithium poisoning. Clin Toxicol 1993; 31: 429-447.

20 Notarianni L. A reassessment of the treatment of salicylate poisoning. Drug Safety 1992; 7: 292-303.

21 Temple AR. Acute and chronic effects of aspirin toxicity and their treatment. Arch Intern Med 1981; 141: 366.

22 Done AK. Aspirin overdose: Incidence, diagnosis and management. Pediatrics 1978; 62(Suppl): 895.

23 Done AK. Salicylate intoxication. Significance of measurements of salicylate in blood in cases of acute ingestion. Pediatrics 1960; 26: 800-807.

24 Hylander B, Kjellstrand CM. Prognostic factors and treatment of severe ethylene glycol intoxication. Int Care Med 1996; 22: 546-552.

25 Pond SM. Manifestations and management of paraquat poisoning. Med J Australia 1990; 152: 256-259.

26 Hart TB, Nevill A, Whitehead A. A statistical approach to the prognostic significance of plasma paraquat concentrations. Lancet 1984; ii: 1222-1223.

27 Rumack BH, Peterson RC, Koch GG, Amara IA. Acetaminophen Overdose: 662 Cases with evaluation of oral acetylcysteine treatment. Arch Intern Med 1981; 141: 380-385. 